\title{
Hydrogen-Induced Step-Edge Roughening of Platinum Electrode Surfaces
}

\author{
Ian T. McCrum, ${ }^{(0)}$ Christoph J. Bondue, and Marc T. M. Koper*(-) \\ Leiden Institute of Chemistry, Leiden University, P.O. Box 9502, 2300 RA Leiden, The Netherlands
}

Supporting Information

ABSTRACT: Electrode surfaces may change their surface structure as a result of the adsorption of chemical species, impacting their catalytic activity. Using density functional theory, we find that the strong adsorption of hydrogen at low electrode potentials promotes the thermodynamics and kinetics of a unique type of roughening of 110-type Pt step edges. This change in surface structure causes the appearance of the so-called "third hydrogen peak" in voltammograms measured on Pt electrodes, an observation that has eluded explanation for over 50 years. Understanding this roughening process is important for structure-sensitive (electro)catalysis and the development of active and stable catalysts.

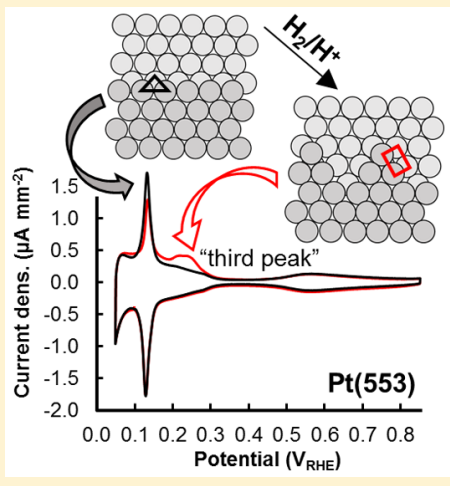

$\mathrm{T}$ he surface structure of a catalyst is important because this structure dictates the activity, selectivity, and mechanism of catalytic reactions occurring on the surface. The binding strength of reactive intermediates to a catalyst surface is dependent on the surface structure and can be correlated to the coordination number of the surface atoms, ${ }^{1,2}$ where atoms with a lower coordination bind adsorbates more strongly. Assuming a reaction that follows a Brønsted-Evans-Polyani (BEP) relation, where the activation energy for an elementary step correlates with the change in free energy of that step, the activation barriers between reactive intermediates will also be dependent on the surface structure. Evidence of surface structure dependence is well known in electrocatalysis, ${ }^{5}$ including for ammonia oxidation, ${ }^{6}$ oxygen reduction, ${ }^{7,8}$ carbon dioxide reduction, ${ }^{9,10}$ ketone reduction, ${ }^{11}$ and methanol oxidation. ${ }^{12}$ Understanding this surface structure dependence is therefore important for designing highly active and selective catalysts.

In studies of structural dependence in electrocatalysis, it is often assumed that the nominal surface does not change during catalysis. However, as the adsorption of spectator and reactive species alters the surface energy of a catalyst surface, and therefore its stability, the surface structure of a catalyst can change under reaction conditions. In the electrochemical environment, cycling the potential, which exposes the catalyst cyclically to oxidizing and reducing conditions, is known to have significant effects on the surface structure. For example, for nanoparticle (NP) Pt, dissolution/deposition and oxide formation/reduction result in Ostwald ripening ${ }^{13}$ and shape changes, where the NP shape depends on cycling conditions. Square-wave cycling of Pt NPs to potentials below $0 \mathrm{~V}_{\mathrm{RHE}}$ results in the formation of tetrahexahedral NPs expressing a high fraction of (100) sites, ${ }^{14}$ in part because the 100 surface is most stable at low potentials in an aqueous electrolyte due to its strong binding of hydrogen. ${ }^{15}$ The cathodic corrosion of $\mathrm{Pt}$ at negative potentials similarly leads to an increase in the exposure of 100 sites. $^{16}$ On a $\mathrm{Pt}(111)$ single-crystal surface, cycling the electrode potential results in the growth of islands exposing a large fraction of (110) step sites, ${ }^{17,18}$ where the 110 step sites have the lowest surface energy at potentials where the surface oxide formed at high potentials is reduced due to the strong binding of $\mathrm{OH}$ and $\mathrm{H}_{2} \mathrm{O}$ to this surface. ${ }^{15}$ This surface restructuring has a direct effect on the electrocatalytic activity. ${ }^{19}$ Each of these structural changes results in a change to the cyclic voltammogram measured on the platinum electrode, as the adsorption of species that correspond to the voltammetric peaks is surface-structure-sensitive. ${ }^{20}$

The (in situ) experimental confirmation of possible structural changes of platinum electrodes typically relies on measuring the blank voltammetry of the electrode in the "hydrogen region". $5,21,22$ Whereas there is a good general understanding of how changes in the hydrogen region relate to structural changes on the surface, the so-called "third hydrogen peak" in cyclic voltammograms measured on platinum electrodes has eluded a detailed explanation. ${ }^{20,23,24}$ This peak in current appears near $0.22 \mathrm{~V}_{\mathrm{RHE}}$, between peaks corresponding to the exchange of adsorbed hydrogen with hydroxide and water on (110) and on (100) step sites (hence the name "third-peak"), in cyclic voltammograms measured on polycrystalline or single-crystalline platinum electrodes after these electrodes are held at high potential (above $\left.\sim 1.3 \mathrm{~V}_{\mathrm{RHE}}\right)^{23-28}$ or low potential (below $\left.0.17 \mathrm{~V}_{\mathrm{RHE}}\right) .^{23-25,29}$ It has been

Received: August 30, 2019

Accepted: October 16, 2019

Published: October 16, 2019 

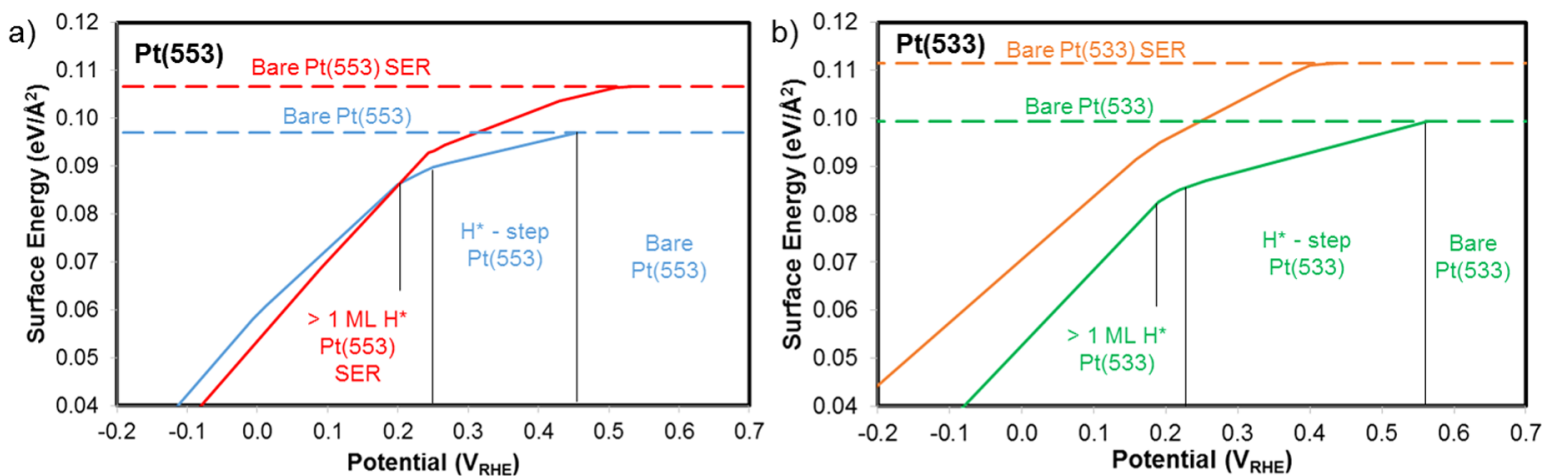

c)
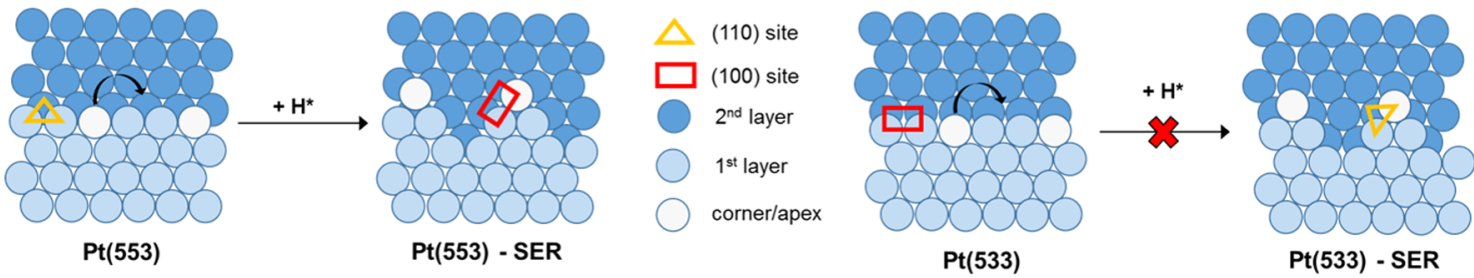

Figure 1. Surface energy of the pristine and roughened (denoted "SER") (a) $\mathrm{Pt}(553)$ and (b) $\mathrm{Pt}(533)$ in the absence (dashed lines) and presence (solid lines) of $\mathrm{H}^{*}$. Each change in slope indicates a change in hydrogen coverage. (c) Step-edge roughening process, where $1 / 3$ of the step atoms are moved along the step edge. This creates a corner/apex site and step sites of the opposite type $(110 \rightarrow 100$ and $100 \rightarrow 110)$. This process is favorable in the presence of adsorbed hydrogen for $\mathrm{Pt}(553)$ (where the 110 sites are converted to 100 sites) but is unfavorable for $\mathrm{Pt}(533)$, even in the presence of $\mathrm{H}^{*}$.

suggested to be due to the formation of a unique surface or subsurface binding site by oxygen (hydrogen) adsorption at high (low) potentials that is then filled by hydrogen at low potentials, $^{24,26,28}$ or formed at low potentials by the absorption/desorption of subsurface atomic hydrogen ${ }^{30,31}$ or subsurface molecular hydrogen. ${ }^{27}$ Work performed with singlecrystal electrodes has shown that the third peak is exclusively related to the presence of (110) step sites, (110) terraces, or $(110)-(2 \times 1)$ domains. ${ }^{23,24,32}$ In situ infrared spectroscopy has provided evidence of the presence of surface-adsorbed hydrogen but not of subsurface hydrogen. ${ }^{23}$

In this Letter, we use density functional theory (DFT) to explain the appearance of the "third peak" after holding the platinum electrode at negative potentials to be due to a change in the surface structure of the platinum electrode. Specifically, the high coverage of hydrogen present at low potentials promotes a particular type of roughening of the (110)-type step edge. We find the strong binding of hydrogen makes the roughening process thermodynamically favorable at potentials below that of the third peak. Furthermore, we find that a high coverage of adsorbed hydrogen makes the roughening process kinetically feasible, lowering the activation energy considerably compared with that calculated for low hydrogen coverage.

This step-edge roughening is important because it affects how the structure-sensitive catalyst behavior is interpreted and illustrates that step sites, which are frequently a source of high catalytic activity, exhibit a structure under reaction conditions that is different from that of the pristine step edge. Active $\mathrm{Pt}$ step sites will be reconstructed under reducing conditions in both the gas phase and electrochemical environments, such as during hydrogen evolution or organic reduction/hydrogenation reactions, meaning that this high activity may actually be due to these reconstructed sites. This change in structure, and how it may positively or negatively affect the activity/ selectivity, cannot be neglected during catalyst design. Furthermore, because the driving force derives from a difference in the adsorbate binding strength between (100) and (110) step types, other adsorbates may similarly drive other metal step edges to roughen.

Surface Energy of Stepped Pt Surfaces at Low Potentials. Figure 1 shows the surface energy of $\operatorname{Pt}(553)$ and $\mathrm{Pt}(533)$ at low potentials in the presence of an increasing coverage of adsorbed hydrogen. The $\operatorname{Pt}(553)$ and $\operatorname{Pt}(533)$ surface are composed of (110)- (synonymous with (111)-type steps; the difference in nomenclature refers to whether the atom at the bottom of the step-edge is considered to be a part of the step or a part of the terrace) and (100)-type steps, respectively, and (111)-like terraces. In the electrochemical environment, prior work has shown that the adsorption of hydrogen on the step edges of Pt occurs with an exchange of adsorbed hydroxide and water; considering this exchange with DFT yields stepassociated peak potentials that match the experiment for both (110) and (100) steps. ${ }^{33-35}$ We neglect hydroxide and water adsorption here and examine only hydrogen adsorption to both the step and terrace sites because hydroxide and water adsorption are not expected to play a role at the potentials where the step-edge roughening occurs. We also neglect the (bi)sulfate adsorption on the (111) terraces (important for the comparison to the experiment in a sulfuric acid electrolyte) because it occurs at potentials $\left(0.35 \text { to } 0.5 \mathrm{~V}_{\mathrm{RHE}}\right)^{36}$ positive of that where the third peak appears $\left(0.22 \mathrm{~V}_{\mathrm{RHE}}\right)$.

As shown in Figure 1, in the absence of hydroxide and water adsorption, both $\mathrm{Pt}(553)$ and $\mathrm{Pt}(533)$ are bare at high potentials (above $\sim 0.6 \mathrm{~V}_{\mathrm{RHE}}$ ). As the potential is decreased, hydrogen adsorption first becomes favorable on the (110) and (100) step edges (predicted to be at $0.46 \mathrm{~V}_{\mathrm{RHE}}$ on $\mathrm{Pt}(553)$ and $0.56 \mathrm{~V}_{\mathrm{RHE}}$ on $\mathrm{Pt}(533)$, although the inclusion of hydroxide adsorption will shift these potentials to somewhat lower values $^{33}$ ) because the platinum atoms at the step edge have a lower coordination number than those at the terrace and therefore bind adsorbates strongly. Adsorption is stronger on the (100) step of $\operatorname{Pt}(533)$ than on the (110) step of $\operatorname{Pt}(553)$. 
The adsorption of hydrogen at both step edges is relatively independent of coverage, ${ }^{33}$ and the steps become fully occupied with hydrogen close to the onset potential for hydrogen adsorption. As the potential further decreases, hydrogen begins to adsorb on the terrace sites. The hydrogen adsorption on the terrace is strongly dependent on coverage, with repulsive interactions between neighboring hydrogen adatoms. ${ }^{37}$ As hydrogen adsorption becomes thermodynamically favorable, the surface energy decreases. As the hydrogen coverage increases, the dependence of the surface energy on the electrode potential becomes greater as a greater number of electrons are transferred per unit area.

Also shown in Figure 1 is the surface energy of $\operatorname{Pt}(553)$ and $\mathrm{Pt}$ (533) in the presence of adsorbed hydrogen, where the step edge has been roughened. A single degree of roughening has been examined, where $1 / 3$ of the step-edge atoms are removed from the step edge and translated along the step edge to the minimum energy adsorption site. This roughening process creates a corner or "apex" site and step sites of the opposite type (converting 110 steps to 100 steps and vice versa) (Figure 1c). This apex site is not a kink, as a kink separates step sites of opposite types (110-kink-100), whereas this apex site separates those of the same type (100-apex-100). Figure 1 illustrates that this process is unfavorable (the roughened step edge has a higher surface energy) in vacuum in the absence of adsorbed hydrogen because forming the under-coordinated corner/apex sites requires breaking strong $\mathrm{Pt}-\mathrm{Pt}$ bonds. It should be noted that all steps exhibit a small degree of roughening at finite temperatures, driven by changes in configurational entropy.

As the potential is lowered, the adsorption of hydrogen onto the roughened step edges becomes favorable compared with the adsorption on straight step edges. For $\mathrm{Pt}(553)$, the potential at which hydrogen adsorbs onto the roughened step edge is more positive (indicating stronger adsorption) than that on the smooth, pristine step edge. This is due to the roughening process producing (1) an apex/corner site composed of a platinum atom with a much lower coordination than those in the pristine step edge, resulting in a stronger binding of adsorbates, and (2) sites that have a structure similar to (100) step sites, which show stronger binding of $\mathrm{H}^{*}$ than (110) step sites. These two factors lead to the roughened $\mathrm{Pt}$ (553) step binding hydrogen more strongly than the pristine $\mathrm{Pt}(553)$ step. Adsorption on the roughened $\mathrm{Pt}(533)$ step is less favorable (less positive onset potential) because on this surface (110) adsorption sites are produced upon roughening, having weaker $\mathrm{H}^{*}$ binding than the original (100) sites.

Below a critical potential of $0.20 \mathrm{~V}_{\mathrm{RHE}}$, the roughened (110) step edge of $\mathrm{Pt}(553)$ becomes more stable than the pristine step edge. Ignoring kinetic considerations, this represents the equilibrium potential where a (110) step edge will roughen. For the $\operatorname{Pt}(533)$ surface, the roughened step edge is never more stable under any of the hydrogen coverages and potentials considered here. Because hydrogen binds more strongly on (100) step sites than on (110) step sites (Figure $1)$, the roughening process on $\operatorname{Pt}(553)$ is favorable in the presence of hydrogen because weak binding 110 sites are converted to stronger binding 100 sites, and the roughening process is unfavorable for $\operatorname{Pt}(533)$ because the strong binding 100 sites are converted to weak binding 110 sites. Even the strong binding on the under-coordinated apex site does not outweigh the weaker binding on the new (110) step edge sites created upon roughening of the step on $\mathrm{Pt}(533)$.
This calculated critical potential $\left(0.20 \mathrm{~V}_{\mathrm{RHE}}\right)$ corresponds well with the experimentally measured location of the third peak $\left(0.17 \mathrm{~V}_{\mathrm{RHE}}\right.$, taken as the average of the positive and negative going scans ${ }^{23}$ ), where for potentials more positive of this potential the step edge is pristine, and below this potential, the step edge roughens. The adsorption of hydroxide and water along the step edge, especially on the roughened step edge, could alter the calculated critical potential. Additionally, experimental work examining the appearance of the third peak on Pt single-crystal electrodes confirms the step-type dependence we have identified here, where the third peak appears only on surfaces containing (110) steps and does not appear after the same treatment procedure (holding at a low potential) on surfaces containing (100) steps. ${ }^{23,24}$ Hydrogen-induced stepedge roughening is also thermodynamically favorable on the 1 $\times 2$ reconstructed $\mathrm{Pt}(110)$ surface, which also contains 110 steps (Figure S1). We leave the more detailed investigation of this roughening process on the $\operatorname{Pt}(110)$ surface and the investigation of the roughening of stepped surfaces with (110) terraces as future work because their complex surface structure leads to a greater possible combination of atom movements, which would result in a similar roughening phenomenon.

The exact potential where the roughened step edge becomes more stable than the pristine step edge, as calculated with DFT, depends to some extent on the exchange-correlation functional chosen; a different functional may yield a potential further or closer to that measured experimentally. As a benchmark relative to the experiment, we have also calculated the adsorption potential of hydrogen at $1 / 9 \mathrm{ML}$ on $\mathrm{Pt}(111)$ using the same functional, yielding $0.37 \mathrm{~V}_{\mathrm{RHE}}$ (with configurational entropy, assuming no interactions, $0.43 \mathrm{~V}_{\mathrm{RHE}}$ ), close to that measured experimentally, $\sim 0.3 \mathrm{~V}_{\mathrm{RHE}} \cdot{ }^{38,39}$

Adsorbate-induced reconstruction is a well-studied phenomenon. (See Somorjai and Van Hove ${ }^{40}$ for a thorough introduction and review.) This includes hydrogen-driven reconstructions, for example, on $\mathrm{Cu}(100)$, which has been studied in both the gas phase ${ }^{41,42}$ and electrochemical environments ${ }^{43,44}$ as well as with DFT. ${ }^{45}$ The reconstruction we have defined here, however, is a specific step reconstruction under electrochemical conditions, where (110)-type sites are converted into a "corner" site and (100)-type sites, which has not been previously reported (to our knowledge). Tao et al. and $\mathrm{Zhu}$ et al. found a similarly shaped but significantly larger and more complex $\mathrm{Pt}$ step reconstruction, driven by the adsorption of strong-binding CO. ${ }^{46,47}$ Additionally, it is the first time a specific reconstruction has been investigated as a cause of the third peak observed in platinum voltammograms. As DFT shows that this process is driven by the stronger binding of hydrogen to (100) sites than (110) sites, this work provides chemical insight into this reconstruction process, suggesting that any adsorbate that shows the same binding preference could drive a similar reconstruction.

Having shown that the hydrogen-induced roughening of (110)-type step edges is thermodynamically favorable, we use DFT to determine if the roughening process is kinetically favorable. There are two main paths for the roughening process: (1) one Pt atom translates across the step edge and (2) a "concerted" mechanism where two neighboring Pt atoms translate together, where the second atom is to fill the hole created by the first. Only results for the concerted mechanism are shown. Figure 2 shows a reaction energy diagram for the roughening process at various hydrogen coverages for the chosen model of the step-edge roughening drawn in Figure 1. 


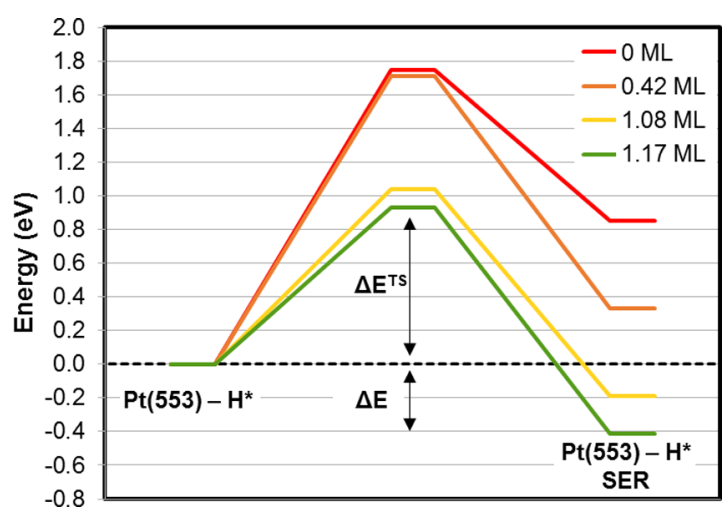

Figure 2. Reaction energy diagram for step-edge roughening of $\operatorname{Pt}(553)$. Activation barriers $\left(\Delta E^{\mathrm{TS}}\right)$ and reaction energies $(\Delta E)$ are calculated in the absence of hydrogen (red), in the presence of a low hydrogen coverage (orange, $0.42 \mathrm{ML} \mathrm{H}^{*}$ ), and in the presence of two high hydrogen coverages (yellow, 1.08 $\mathrm{ML} \mathrm{H}^{*}$; green, 1.17 $\mathrm{ML} \mathrm{H}^{*}$ ). Each reaction path is referenced to the $\mathrm{Pt}(553)$ surface at the indicated hydrogen coverage for clarity. At the highest hydrogen coverage examined $(1.17 \mathrm{ML})$, the reaction energy is $-0.41 \mathrm{eV}$ and the activation energy is $0.93 \mathrm{eV}$.

In the absence of hydrogen (in vacuum), the roughening process is thermodynamically unfavorable with a high kinetic barrier; the activation energy is $1.75 \mathrm{eV}$. The rate of this process at room temperature would be exceedingly slow. Activation energies for typical electrocatalytic reactions at room temperature are between 0.2 and $1.4 \mathrm{eV}^{48-51}$ With the step edge fully occupied by hydrogen, the reaction energy decreases, although the activation energy is still large at 1.71 $\mathrm{eV}$. However, as the hydrogen coverage is further increased, the reaction energy becomes negative (thermodynamically favorable), and the activation energy significantly decreases (becoming kinetically favorable). At the highest hydrogen coverage examined, the activation energy is $0.93 \mathrm{eV}$. A reaction with this activation energy would yield a measurable rate at room temperature (measurable on the time scale of common cyclic voltammetric scan rates on the order of $0.05 \mathrm{~V} / \mathrm{s}$ ) and is comparable to that experimentally measured for $\mathrm{CO}$ oxidation on platinum surfaces (with a barrier of 0.9 to $1.4 \mathrm{eV}$ ). ${ }^{49,51}$ Animations illustrating the reaction paths and images of the initial, transition, and final states (Figures S2-S5) are given in the Supporting Information.

Figure 2 illustrates that the presence of a high coverage of adsorbed hydrogen can lower the barrier for platinum stepedge roughening by $>0.8 \mathrm{eV}$ (resulting in an increase in the rate by a factor of $8.5 \times 10^{13}$ relative to that in the absence of hydrogen). Previous work by Besenbacher et al. has shown that a single hydrogen adatom can lower the activation energy for platinum adatom diffusion by $0.09 \mathrm{eV}^{52} \mathrm{Zhu}$ et al. have observed increases in the noise level in high-pressure scanning tunneling microscopy (STM) images with increasing hydrogen pressure believed to be due to increased step and kink atom mobility (with increasing hydrogen pressure). ${ }^{53}$ The reduction in activation energy follows a BEP relation for the roughening process; as the hydrogen coverage increases, the reaction energy becomes more negative, resulting in a decrease in the activation energy. Such a relation can be explained considering bond-order conservation, where increased bonding to hydrogen adatoms (by platinum step-edge atoms) results in weaker bonding with the rest of the platinum surface.
We would like to point out that other mechanisms for the roughening process could exist, including those involving the simultaneous adsorption/desorption of hydrogen with the roughening/deroughening of the step edge. The reaction paths we have examined here represent a possible mechanism that yields, at least in the presence of a high coverage of hydrogen, an activation barrier that is surmountable (resulting in a measurable rate of reaction at room temperature). More favorable reaction paths would yield lower barriers and therefore higher reaction rates.

Comparison with Experiment. The anomalous "third peak" appears at a potential of $0.22 \mathrm{~V}_{\mathrm{RHE}}$ in the positive going scan of cyclic voltammograms at a scan rate of $0.05 \mathrm{~V} / \mathrm{s}^{23}$ The cathodic portion of this peak appears near $0.125 \mathrm{~V}_{\mathrm{RHE}}$ at the same scan rate and is difficult to see because it is lumped in with the peak corresponding to the exchange of hydroxide and water with hydrogen on the (110) step. ${ }^{23}$ This significant peak splitting suggests that the process that produces the third peak is kinetically slow. $^{23,29}$ The thermodynamics calculated here for the step-edge roughening process indicate that it becomes favorable at a potential of $0.2 \mathrm{~V}_{\mathrm{RHE}}$ (Figure 1), matching well with the experimentally measured location, taken as an average of the location of the peak in the positive and negative going scans $\left(0.17 \mathrm{~V}_{\mathrm{RHE}}\right)$. Furthermore, the large DFT calculated barrier $(0.93 \mathrm{eV})$ is consistent with a kinetically slow reaction, on the time scale of a $0.05 \mathrm{~V} / \mathrm{s}$ scan rate. However, a detailed comparison of our DFT kinetics to experiment requires temperature-dependent studies, so that the activation energy for step-edge roughening can be explicitly measured.

After a single, or a few, cycle(s) positive of the potential where the third peak appears, the peak disappears and does not return unless the potential is held below $0.17 \mathrm{~V}_{\mathrm{RHE}}{ }^{23}$ This implies that the process that causes the third peak is structurally reversible, consistent with the step-edge roughening examined here, which occurs with only a small number of step-edge atoms diffusing a short distance along the step edge. As mentioned, the third peak is present only on electrodes containing (110) steps, ${ }^{23,24}$ matching our finding that this step-edge roughening process is not favorable (at least with $\mathrm{H}^{*}$ ) on (100)-type step edges. Lastly, the ratio of the charge lost in the step-associated peak (upon the appearance of the third peak) to that gained in the third peak measured experimentally $(1: 2)^{23,29}$ matches the degree of step-edge roughening we have examined here, where the movement of one step atom creates two new step sites (which contribute to the third peak).

We therefore believe that the roughening of (110) step edges explains the appearance of the third peak; the deroughening of the step edge coincident with the desorption of hydrogen adsorbed on the roughened sites is responsible for the anodic portion of the "third peak". This explanation is consistent with the peak being lost after a single cycle through this potential region, as the step relaxes back to the straight, pristine step edge. Furthermore, we can additionally rule out any reconstruction processes that would alter the step density, such as step doubling, because this does not result in the appearance of an additional peak and would only alter the ratio of step to terrace sites. ${ }^{54}$

We note that both anodic (holding at high potentials, $>1$ $\mathrm{V}_{\mathrm{RHE}}$ ) and cathodic (holding at low potentials) treatments can lead to the appearance of the same third peak, but it takes more than one cycle to potentials positive of where the third peak occurs to remove this peak after the anodic treatment. We 

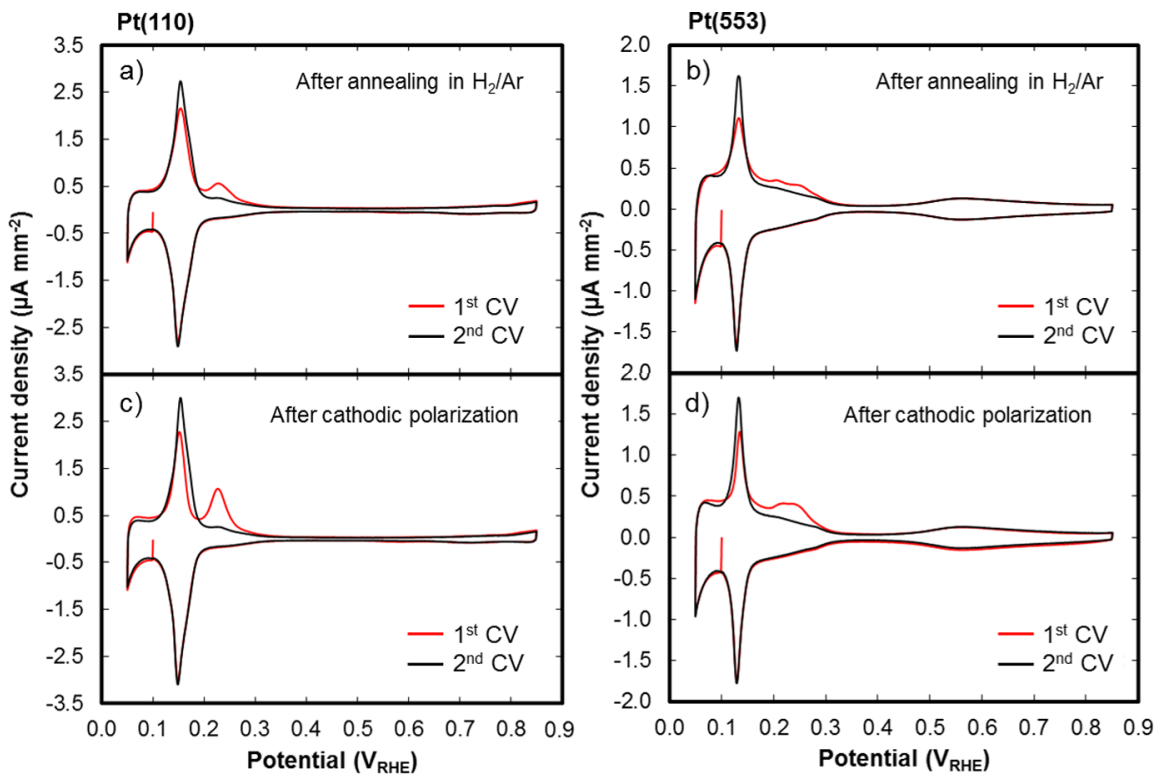

Figure 3. Cyclic voltammograms measured on (a,c) Pt(110) and (b,d) Pt(553) showing the first (red) and second cycles (black) after annealing and cooling in a $\mathrm{H}_{2}+\mathrm{Ar}$ atmosphere $(\mathrm{a}, \mathrm{b})$, after holding at $-0.2 \mathrm{~V}_{\mathrm{RHE}}$ for $60 \mathrm{~s}(\mathrm{c})$, and after holding at $-0.3 \mathrm{~V}_{\mathrm{RHE}}$ for $120 \mathrm{~s}$ (d). The $1 \mathrm{st}$ voltammogram after annealing and cooling clearly shows the third peak, indicating that the step edge was roughened in the hydrogen-containing cooling atmosphere.

can hypothesize that a similar process (step roughening) occurs under anodic conditions but to a greater extent (greater degree of roughening). This anodic treatment, however, is more complex and leads to more extensive surface roughening, including of terraces, resulting in island formation. ${ }^{17}$ Therefore, we limit the discussion here to the effects of the cathodic treatment and roughening of the step induced by adsorbed hydrogen.

Because we find the third peak to be due to step-edge roughening caused by the adsorption of hydrogen (from protons in solution), we might expect similar roughening to occur upon the adsorption of hydrogen from the gas phase. Figure 3 shows the first cyclic voltammograms measured on $\mathrm{Pt}(110)$ and $\mathrm{Pt}(553)$ after postannealing cooling in a hydrogen-containing atmosphere. The third peak is clearly present (Figure 3a,b), indicating that the step edge was roughened by hydrogen present in the annealing/cooling atmosphere and matches that seen on $\mathrm{Pt}(110)$ and $\mathrm{Pt}(553)$ after holding at low potentials $\left(-0.2 \mathrm{~V}_{\mathrm{RHE}}, \mathrm{Pt}(110) ;-0.3\right.$ $\mathrm{V}_{\mathrm{RHE}}, \operatorname{Pt}(553)$ ) (Figure 3c,d).

This step reconstruction/roughening process warrants further investigation, especially with in situ imaging techniques, such as electrochemical scanning tunneling microscopy (ECSTM), to see if the structure of the step can be visualized under reaction conditions. We expect such a measurement to be difficult, however, because the relatively small structure formed upon roughening (with one atom translating along the step edge to produce the "corner" site) requires imaging with atomic resolution in the electrochemical environment. Wei et al. imaged $\mathrm{Pt}(332)$ and $\mathrm{Pt}(997)$ (stepped platinum electrodes with (110)-type steps) in the electrochemical environment with EC-STM at $0.1 \mathrm{~V}_{\mathrm{RHE}}$ (where we would expect this roughening process to be slow but favorable) and observed a high density of kink sites and commented that under the high coverage of hydrogen, high-resolution STM imaging revealed only poorly defined step-edge structures. ${ }^{55}$ This is in contrast with STM images taken at the same potential but in the presence of strongly adsorbed $\mathrm{CO}$, where straight, defect-free step edges were clearly seen. ${ }^{55}$ Even in the gas-phase (nonelectrochemical) environment, high-resolution STM by Zhu et al. on $\mathrm{Pt}$ (557) in the presence of $100 \mathrm{mTorr}$ and 1 Torr of $\mathrm{H}_{2}$ yields images that show "frizzy" and blurred steps (respectively), presumably due to increased step and kink atom mobility in the presence of high coverages of adsorbed hydrogen and increased noise from rapid hydrogen adsorption and desorption. ${ }^{53}$

This roughening process changes the structure of the step edge, which has significant implications in surface science and catalysis. Because the roughening is favorable under a high coverage of adsorbed hydrogen, any catalytic reaction carried out under reducing conditions in the gas phase, liquid phase, or electrochemical environment on a platinum surface may be occurring on a surface with roughened (110) step edges. This affects how we interpret structure-dependent catalysis: If a surface with nominal (110) step edges exhibits a particular activity and selectivity for a given reaction, then we have to consider that the step geometry under the reaction conditions may not be that of the pristine (110) step edge. This is important for understanding how the structure impacts the kinetics and mechanism of the reaction and for the atomisticscale modeling of the reaction. Furthermore, the roughened step edge may have a different stability than the pristine step edge, promoting additional changes to the surface structure. Reactions carried out under reducing conditions include organic reductions/hydrogenations and electrochemical hydrogen evolution. There exist many cases in prior literature where these reactions have been carried out on platinum surfaces that show the third peak (suggesting that the (110) step edges were roughened at low potentials), including during $\mathrm{CO}_{2}$ electroreduction, ${ }^{56,57}$ acetone reduction, ${ }^{58} \mathrm{~m}$-xylene reduction, ${ }^{59} \mathrm{p}$ dihydroxybenzene reduction, ${ }^{60}$ hydroquinone reduction, ${ }^{61}$ and hydrogen evolution. ${ }^{62}$

Subsurface Hydrogen. The absorption of subsurface hydrogen (below the first atomic layer) on $\operatorname{Pt}(553)$ and $\operatorname{Pt}(110)$ in the 
absence and presence of varying coverages of surface-adsorbed hydrogen was also examined with DFT. The absorption of hydrogen into $\mathrm{Pt}(553)$ and $\mathrm{Pt}(110)$ is favorable only at very negative potentials, -0.34 and $-0.72 \mathrm{~V}_{\mathrm{RHE}}$, respectively. This rules out the involvement of subsurface hydrogen in the appearance of the third peak. Furthermore, achieving a similar hydrogen chemical potential in the gas phase (as would be necessary to produce the third peak seen in voltammograms measured after postannealing cooling in hydrogen if subsurface hydrogen were responsible) would require a hydrogen pressure of $10^{12}$ to $10^{24}$ atm.

The presence of surface-adsorbed hydrogen promotes the absorption of subsurface hydrogen on $\mathrm{Pt}(110)$ (Figure S1; by $-0.46 \mathrm{eV}$ in the presence of $2 \mathrm{ML} \mathrm{H}^{*}$ on $\left.\mathrm{Pt}(110) 1 \times 1\right)$; however, the adsorption of higher coverages of hydrogen on the surface (of both $\mathrm{Pt}(553)$ and $\mathrm{Pt}(110)$ ) is always more favorable than subsurface absorption. Even in the presence of this high coverage of adsorbed hydrogen, the formation of subsurface hydrogen occurs at potentials well below where the third peak appears experimentally. Future work should use DFT to examine the subsurface hydrogen formation at lower concentrations and on defected $\mathrm{Pt}(110)$ surfaces as well as the roughened step edges on $\mathrm{Pt}(110)$ with fewer step atoms displaced per unit area (smaller degree of roughening).

In summary, holding a platinum electrode containing (110) step sites at low potentials (below $0.17 \mathrm{~V}_{\mathrm{RHE}}$ ) results in the appearance of the anomalous "third peak" at $0.22 \mathrm{~V}_{\mathrm{RHE}}$ in the cyclic voltammogram measured on the same electrode. The strong binding of hydrogen to $\mathrm{Pt}$ step sites and the stronger binding on (100) step sites relative to (110)-type step sites promotes a unique roughening of the (110) step at potentials near where the anomalous third peak occurs, where (110) step sites are converted to an under-coordinated "corner" site and (100) step sites. Higher coverages of hydrogen (adsorbed at lower potentials) make the roughening process kinetically favorable, lowering the activation energy by $0.8 \mathrm{eV}$ relative to that at low coverage. We believe this step-edge roughening explains the appearance of the third peak, where the deroughening of the step edge coincident with hydrogen desorption results in the anodic portion of the peak. The absorption/desorption of subsurface hydrogen is ruled out as a cause of the third peak because it is significantly less thermodynamically favorable.

We additionally find experimental evidence supporting the notion that gas-phase hydrogen can similarly drive the stepedge roughening process (important because the thermodynamics are the same for 1 atm hydrogen gas and for $1 \mathrm{M}$ protons at $0 \mathrm{~V}_{\mathrm{RHE}}$ in the electrochemical environment). Cyclic voltammograms measured on $\mathrm{Pt}(110)$ and $\mathrm{Pt}(553)$ after annealing and cooling of the crystal in a hydrogen-containing atmosphere show the presence of the third peak.

Understanding the mechanism and driving forces for stepedge roughening is important for knowing the structure of the catalyst surface under reducing reaction conditions. This work suggests that active $\mathrm{Pt}$ (step) sites are reconstructed under reducing conditions in the gas-phase, aqueous, and electrochemical environments, including, for example, during hydrogen evolution or organic hydrogenation/reduction reactions, suggesting that the measured activity may be due to these reconstructed sites. Interpreting correlations between surface structure and activity therefore require the consideration of this roughening process. Highly active and selective catalysts can be designed only if the surface structure under reaction conditions is known. Furthermore, the surface structure and surface energy under reaction conditions determine the catalyst stability. Understanding step-edge thermodynamics and roughening gives insight into the long-term catalyst behavior. We expect any adsorbate that, like hydrogen on platinum, shows a difference in binding energy between (110)and (100)-type step sites for a particular metal surface to provide a driving force for possible step roughening and surface reconstruction.

\section{ASSOCIATED CONTENT}

\section{Supporting Information}

The Supporting Information is available free of charge on the ACS Publications website at DOI: 10.1021/acs.jpclett.9b02544.

Detailed computational and experimental methods, thermodynamics of $\mathrm{Pt}(110)$ step-edge roughening, and images of initial, transition, and final states of the roughening process (PDF)

Animations of the reaction paths for the roughening process (ZIP)

\section{AUTHOR INFORMATION}

\section{Corresponding Author}

*E-mail: m.koper@lic.leidenuniv.nl. ORCID

Ian T. McCrum: 0000-0003-2104-032X

Marc T. M. Koper: 0000-0001-6777-4594

Notes

The authors declare no competing financial interest.

\section{ACKNOWLEDGMENTS}

This project has received funding from the European Union's Horizon 2020 research and innovation programme under the Marie Skłodowska-Curie grant agreement no. 707404. The use of supercomputing facilities at SURFsara was sponsored by NWO Physical Sciences, with financial support by NWO.

\section{REFERENCES}

(1) Calle-Vallejo, F.; Loffreda, D.; Koper, M. T. M.; Sautet, P. Introducing Structural Sensitivity into Adsorption-Energy Scaling Relations by Means of Coordination Numbers. Nat. Chem. 2015, 7, 403.

(2) Calle-Vallejo, F.; Tymoczko, J.; Colic, V.; Vu, Q. H.; Pohl, M. D.; Morgenstern, K.; Loffreda, D.; Sautet, P.; Schuhmann, W.; Bandarenka, A. S. Finding Optimal Surface Sites on Heterogeneous Catalysts by Counting Nearest Neighbors. Science 2015, 350, 185189

(3) Evans, M. G.; Polanyi, M. Inertia and Driving Force of Chemical Reactions. Trans. Faraday Soc. 1938, 34, 11-24.

(4) Bligaard, T.; Nørskov, J. K.; Dahl, S.; Matthiesen, J.; Christensen, C. H.; Sehested, J. The Brønsted-Evans-Polanyi Relation and the Volcano Curve in Heterogeneous Catalysis. J. Catal. 2004, 224, 206217.

(5) Koper, M. T. M. Structure Sensitivity and Nanoscale Effects in Electrocatalysis. Nanoscale 2011, 3, 2054-2073.

(6) Vidal-Iglesias, F. J.; García-Aráez, N.; Montiel, V.; Feliu, J. M.; Aldaz, A. Selective Electrocatalysis of Ammonia Oxidation on $\mathrm{Pt}(100)$ Sites in Alkaline Medium. Electrochem. Commun. 2003, 5, 22-26.

(7) Marković, N. M.; Adžić, R. R.; Cahan, B. D.; Yeager, E. B. Structural Effects in Electrocatalysis: Oxygen Reduction on Platinum Low Index Single-Crystal Surfaces in Perchloric Acid Solutions. J. Electroanal. Chem. 1994, 377, 249-259. 
(8) Marković, N. M.; Gasteiger, H. A.; Ross, P. N. Oxygen Reduction on Platinum Low-Index Single-Crystal Surfaces in Alkaline Solution: Rotating Ring Disk Pt(Hkl) Studies. J. Phys. Chem. 1996, 100, 6715-6721.

(9) Hori, Y.; Takahashi, I.; Koga, O.; Hoshi, N. Selective Formation of $\mathrm{C} 2$ Compounds from Electrochemical Reduction of $\mathrm{CO} 2$ at a Series of Copper Single Crystal Electrodes. J. Phys. Chem. B 2002, 106, 15-17.

(10) Schouten, K. J. P.; Qin, Z.; Pérez Gallent, E.; Koper, M. T. M. Two Pathways for the Formation of Ethylene in CO Reduction on Single-Crystal Copper Electrodes. J. Am. Chem. Soc. 2012, 134, 98649867.

(11) Bondue, C. J.; Calle-Vallejo, F.; Figueiredo, M. C.; Koper, M. T. M. Structural Principles to Steer the Selectivity of the Electrocatalytic Reduction of Aliphatic Ketones on Platinum. Nat. Catal. 2019, 2, 243-250.

(12) Housmans, T. H. M.; Wonders, A. H.; Koper, M. T. M. Structure Sensitivity of Methanol Electrooxidation Pathways on Platinum: An On-Line Electrochemical Mass Spectrometry Study. J. Phys. Chem. B 2006, 110, 10021-10031.

(13) Shao-Horn, Y.; Sheng, W. C.; Chen, S.; Ferreira, P. J.; Holby, E. F.; Morgan, D. Instability of Supported Platinum Nanoparticles in Low-Temperature Fuel Cells. Top. Catal. 2007, 46, 285-305.

(14) Tian, N.; Zhou, Z.-Y.; Sun, S.-G.; Ding, Y.; Wang, Z. L. Synthesis of Tetrahexahedral Platinum Nanocrystals with High-Index Facets and High Electro-Oxidation Activity. Science 2007, 316, 732735.

(15) McCrum, I. T.; Hickner, M. A.; Janik, M. J. First-Principles Calculation of Pt Surface Energies in an Electrochemical Environment: Thermodynamic Driving Forces for Surface Faceting and Nanoparticle Reconstruction. Langmuir 2017, 33, 7043-7052.

(16) Hersbach, T. J. P.; Yanson, A. I.; Koper, M. T. M. Anisotropic Etching of Platinum Electrodes at the Onset of Cathodic Corrosion. Nat. Commun. 2016, 7, 12653.

(17) Jacobse, L.; Huang, Y.-F.; Koper, M. T. M.; Rost, M. J. Correlation of Surface Site Formation to Nanoisland Growth in the Electrochemical Roughening of Pt(111). Nat. Mater. 2018, 17, 277282.

(18) Wakisaka, M.; Asizawa, S.; Uchida, H.; Watanabe, M. In Situ STM Observation of Morphological Changes of the $\mathrm{Pt}(111)$ Electrode Surface During Potential Cycling in $10 \mathrm{mM}$ HF Solution. Phys. Chem. Chem. Phys. 2010, 12, 4184-4190.

(19) Löffler, T.; Bussar, R.; Xiao, X.; Ernst, S.; Baltruschat, H. The Adsorption of Ethene on Vicinally Stepped Electrode Surfaces and the Effect of Temperature. J. Electroanal. Chem. 2009, 629, 1-14.

(20) Will, F. G. Hydrogen Adsorption on Platinum Single Crystal Electrodes: I. Isotherms and Heats of Adsorption. J. Electrochem. Soc. 1965, 112, 451-455.

(21) Solla-Gullón, J.; Rodríguez, P.; Herrero, E.; Aldaz, A.; Feliu, J. M. Surface Characterization of Platinum Electrodes. Phys. Chem. Chem. Phys. 2008, 10, 1359-1373.

(22) Vidal-Iglesias, F. J.; Arán-Ais, R. M.; Solla-Gullón, J.; Herrero, E.; Feliu, J. M. Electrochemical Characterization of Shape-Controlled Pt Nanoparticles in Different Supporting Electrolytes. ACS Catal. 2012, 2, 901-910.

(23) Diaz-Morales, O.; Hersbach, T. J. P.; Badan, C.; Garcia, A. C.; Koper, M. T. M. Hydrogen Adsorption on Nano-Structured Platinum Electrodes. Faraday Discuss. 2018, 210, 301-315.

(24) Gómez, R.; Clavilier, J. Electrochemical Behaviour of Platinum Surfaces Containing (110) Sites and the Problem of the Third Oxidation Peak. J. Electroanal. Chem. 1993, 354, 189-208.

(25) Woodard, F. E.; Scortichini, C. L.; Reilley, C. N. Hydrogen Chemisorption and Related Anion Effects on $\mathrm{Pt}(110)$ Electrodes. J. Electroanal. Chem. Interfacial Electrochem. 1983, 151, 109-131.

(26) Loo, B. H.; Furtak, T. E. Intrinsic Heterogeneity in the Multiple States of Adsorbed Hydrogen on Polycrystalline Platinum. Electrochim. Acta 1980, 25, 505-508.
(27) Frelink, T.; Visscher, W.; van Veen, J. A. R. The Third Anodic Hydrogen Peak on Platinum; Subsurface H2 Adsorption. Electrochim. Acta 1995, 40, 545-549.

(28) Berkes, B. B.; Inzelt, G. Generation and Electrochemical Nanogravimetric Response of the Third Anodic Hydrogen Peak on a Platinum Electrode in Sulfuric Acid Media. J. Solid State Electrochem. 2014, 18, 1239-1249.

(29) Sumino, M. P.; Shibata, S. Specific Adsorption of Hydrogen on Polycrystalline Platinum Electrode. Electrochim. Acta 1992, 37, 26292635.

(30) Breiter, M. W. Electrochemical Processes in Fuel Cells; Springer: New York, 1969.

(31) Martins, M. E.; Zinola, C. F.; Arvia, A. J. Voltammetric Response of Hydrogen Adsorbates on Platinum in Acid Solutions: A Possible H-Adatom Subsurface State. J. Braz. Chem. Soc. 1997, 8, 363-370.

(32) Souza-Garcia, J.; Angelucci, C. A.; Climent, V.; Feliu, J. M. Electrochemical Features of $\mathrm{Pt}(\mathrm{S})[\mathrm{N}(110) \times(100)]$ Surfaces in Acidic Media. Electrochem. Commun. 2013, 34, 291-294.

(33) McCrum, I. T.; Janik, M. J. First Principles Simulations of Cyclic Voltammograms on Stepped $\mathrm{Pt}(553)$ and $\mathrm{Pt}(533)$ Electrode Surfaces. ChemElectroChem 2016, 3, 1609-1617.

(34) Chen, X.; McCrum, I. T.; Schwarz, K. A.; Janik, M. J.; Koper, M. T. M. Co-Adsorption of Cations as the Cause of the Apparent pH Dependence of Hydrogen Adsorption on a Stepped Platinum SingleCrystal Electrode. Angew. Chem., Int. Ed. 2017, 56, 15025-15029.

(35) McCrum, I. T.; Chen, X.; Schwarz, K. A.; Janik, M. J.; Koper, M. T. M. Effect of Step Density and Orientation on the Apparent pH Dependence of Hydrogen and Hydroxide Adsorption on Stepped Platinum Surfaces. J. Phys. Chem. C 2018, 122, 16756-16764.

(36) Mostany, J.; Herrero, E.; Feliu, J. M.; Lipkowski, J. Thermodynamic Studies of Anion Adsorption at Stepped Platinum(Hkl) Electrode Surfaces in Sulfuric Acid Solutions. J. Phys. Chem. B 2002, 106, 12787-12796.

(37) Zolfaghari, A.; Jerkiewicz, G. Temperature-Dependent Research on $\mathrm{Pt}(111)$ and $\mathrm{Pt}(100)$ Electrodes in Aqueous H2SO4. J. Electroanal. Chem. 1999, 467, 177-185.

(38) Marković, N. M.; Schmidt, T. J.; Grgur, B. N.; Gasteiger, H. A.; Behm, R. J.; Ross, P. N. Effect of Temperature on Surface Processes at the $\mathrm{Pt}(111)$-Liquid Interface: Hydrogen Adsorption, Oxide Formation, and CO Oxidation. J. Phys. Chem. B 1999, 103, 85688577.

(39) Gómez, R.; Orts, J. M.; Álvarez-Ruiz, B.; Feliu, J. M. Effect of Temperature on Hydrogen Adsorption on $\mathrm{Pt}(111), \mathrm{Pt}(110)$, and $\mathrm{Pt}(100)$ Electrodes in 0.1 M Hclo4. J. Phys. Chem. B 2004, 108, 228238

(40) Somorjai, G. A.; Van Hove, M. A. Adsorbate-Induced Restructuring of Surfaces. Prog. Surf. Sci. 1989, 30, 201-231.

(41) Chorkendorff, I.; Rasmussen, P. B. Reconstruction of $\mathrm{Cu}(100)$ by Adsorption of Atomic Hydrogen. Surf. Sci. 1991, 248, 35-44.

(42) Foss, M.; Besenbacher, F.; Klink, C.; Stensgaard, I. DeuteriumInduced Restructuring of $\mathrm{Cu}(100)$. Chem. Phys. Lett. 1993, 215, 535540.

(43) Matsushima, H.; Haak, C.; Taranovskyy, A.; Gründer, Y.; Magnussen, O. M. In Situ Video STM Studies of the HydrogenInduced Reconstruction of $\mathrm{Cu}(100)$ : Potential and $\mathrm{pH}$ Dependence. Phys. Chem. Chem. Phys. 2010, 12, 13992-13998.

(44) Matsushima, H.; Taranovskyy, A.; Haak, C.; Gründer, Y.; Magnussen, O. M. Reconstruction of $\mathrm{Cu}(100)$ Electrode Surfaces During Hydrogen Evolution. J. Am. Chem. Soc. 2009, 131, 1036210363.

(45) Hellman, A.; Svensson, K.; Andersson, S. Hydrogen-Induced Reconstruction of $\mathrm{Cu}(100)$ : Two-Dimensional and One-Dimensional Structures of Surface Hydride. J. Phys. Chem. C 2014, 118, 1577315778.

(46) Tao, F.; Dag, S.; Wang, L.-W.; Liu, Z.; Butcher, D. R.; Bluhm, H.; Salmeron, M.; Somorjai, G. A. Break-up of Stepped Platinum Catalyst Surfaces by High CO Coverage. Science 2010, 327, 850-853. 
(47) Zhu, Z.; et al. Influence of Step Geometry on the Reconstruction of Stepped Platinum Surfaces under Coadsorption of Ethylene and CO. J. Phys. Chem. Lett. 2014, 5, 2626-2631.

(48) Paulus, U. A.; Wokaun, A.; Scherer, G. G.; Schmidt, T. J.; Stamenkovic, V.; Radmilovic, V.; Markovic, N. M.; Ross, P. N. Oxygen Reduction on Carbon-Supported $\mathrm{Pt}-\mathrm{Ni}$ and $\mathrm{Pt}-\mathrm{Co}$ Alloy Catalysts. J. Phys. Chem. B 2002, 106, 4181-4191.

(49) Farias, M. J. S.; Herrero, E.; Feliu, J. M. Site Selectivity for CO Adsorption and Stripping on Stepped and Kinked Platinum Surfaces in Alkaline Medium. J. Phys. Chem. C 2013, 117, 2903-2913.

(50) Durst, J.; Simon, C.; Hasché, F.; Gasteiger, H. A. Hydrogen Oxidation and Evolution Reaction Kinetics on Carbon Supported Pt, $\mathrm{Ir}, \mathrm{Rh}$, and Pd Electrocatalysts in Acidic Media. J. Electrochem. Soc. 2015, 162, F190-F203.

(51) Herrero, E.; Feliu, J. M.; Blais, S.; Radovic-Hrapovic, Z.; Jerkiewicz, G. Temperature Dependence of CO Chemisorption and Its Oxidative Desorption on the $\mathrm{Pt}(111)$ Electrode. Langmuir 2000, $16,4779-4783$.

(52) Horch, S.; Lorensen, H. T.; Helveg, S.; Lægsgaard, E.; Stensgaard, I.; Jacobsen, K. W.; Nørskov, J. K.; Besenbacher, F. Enhancement of Surface Self-Diffusion of Platinum Atoms by Adsorbed Hydrogen. Nature 1999, 398, 134.

(53) Zhu, Z.; Melaet, G.; Axnanda, S.; Alayoglu, S.; Liu, Z.; Salmeron, M.; Somorjai, G. A. Structure and Chemical State of the Pt(557) Surface During Hydrogen Oxidation Reaction Studied by in Situ Scanning Tunneling Microscopy and X-Ray Photoelectron Spectroscopy. J. Am. Chem. Soc. 2013, 135, 12560-12563.

(54) Clavilier, J.; Rodes, A. Electrochemical Detection and Characterization at $\mathrm{Pt}(\mathrm{N}, \mathrm{N}, \mathrm{N}$ - 2) Oriented Electrodes of Multiatomic Step Formation Induced by Quenching at High Temperatures. J. Electroanal. Chem. 1993, 348, 247-264.

(55) Wei, J.; Liao, W.-c.; Lei, J.; Yau, S.; Chen, Y.-X. Electrified Interfaces of $\mathrm{Pt}(332)$ and $\mathrm{Pt}(997)$ in Acid Containing CO and $\mathrm{KI}$ : As Probed by in Situ Scanning Tunneling Microscopy. J. Phys. Chem. C 2018, 122, 26111-26119.

(56) Smolinka, T.; Heinen, M.; Chen, Y. X.; Jusys, Z.; Lehnert, W.; Behm, R. J. CO2 Reduction on Pt Electrocatalysts and Its Impact on $\mathrm{H} 2$ Oxidation in CO2 Containing Fuel Cell Feed Gas - a Combined in Situ Infrared Spectroscopy, Mass Spectrometry and Fuel Cell Performance Study. Electrochim. Acta 2005, 50, 5189-5199.

(57) Brisard, G. M.; Camargo, A. P. M.; Nart, F. C.; Iwasita, T. OnLine Mass Spectrometry Investigation of the Reduction of Carbon Dioxide in Acidic Media on Polycrystalline Pt. Electrochem. Commun. 2001, 3, 603-607.

(58) Bänsch, B.; Härtung, T.; Baltruschat, H.; Heitbaum, J. Reduction and Oxidation of Adsorbed Acetone at Platinum Electrodes Studied by Dems. J. Electroanal. Chem. Interfacial Electrochem. 1989, 259, 207-215.

(59) Quiroz, M. A.; Córdova, F.; Lamy-Pitara, E.; Barbier, J. Electrocatalytic Hydrogenation of M-Xylene on Platinized-Platinum Electrodes. Electrochim. Acta 2000, 45, 4291-4298.

(60) Sanabria-Chinchilla, J.; Soriaga, M. P.; Bussar, R.; Baltruschat, H. A Dems Study of the Electrocatalytic Hydrogenation and Oxidation of P-Dihydroxybenzene at Polycrystalline and Monocrystalline Platinum Electrodes. J. Appl. Electrochem. 2006, 36, 1253-1260.

(61) Mebrahtu, T.; Berry, G. M.; Soriaga, M. P. The Influence of Orientation on the Electrocatalytic Hydrogenation of Hydroquinone Chemisorbed at Smooth Polycrystalline Platinum Electrodes. J. Electroanal. Chem. Interfacial Electrochem. 1988, 239, 375-386.

(62) Sheng, W.; Zhuang, Z.; Gao, M.; Zheng, J.; Chen, J. G.; Yan, Y. Correlating Hydrogen Oxidation and Evolution Activity on Platinum at Different $\mathrm{pH}$ with Measured Hydrogen Binding Energy. Nat. Commun. 2015, 6, 5848. 